\title{
Outliers - robust ECM cointegration tests based on the trend components
}

\author{
Miguel A. Arranz ${ }^{1}$, Alvaro Escribano ${ }^{2}$ \\ 1 U.D. Matemáticas y Estadística, EUIT Forestal, Universidad Poltécnica de Madrid, \\ Cindad Universitaria, S/n, 28040, Madrid, Spain (e-mail: maarranz@forestales.upm..es) \\ 2 Departamento de Economía, Universidad Carlos III de Madrid, Madrid 126, \\ Getafe (Madrid) 28903, Spain (e-mail: alvaroe@eco.uc3m.es)
}

\begin{abstract}
The main goal of this paper is to analyze the behavior of the ECM noncointegration test when there are additive outliers in the time series under different co-breaking situations. We show that the critical values of the usual ECM test are not robust to the presence of transitory shocks and we suggest a procedure based on signal extraction to bypass this problem. These procedure renders ECM tests with a left tail of distribution under the null that is robust to the presence of additive outliers in the series. The small sample critical values and the empirical power of the test are analyzed by Monte Carlo simulations for several low frequency filters. The proposed empirical methodology is applied to the CPI-based US/Finland real exchange rate.
\end{abstract}

JEL Classification: C22, C12, C15, C52, C51

Key words: Outliers, transitory co-breaks, cointegration testing, trend-component error correction models

\footnotetext{
We thank A. Lucas for kindly providing us with the data for the empirical example. Previous drafts of this paper have greatly improved thanks to the comments of two anonymous referees and the Associate Editor. The first author wishes to thank members of Department of Statistics and Econometrics, Universidad Carlos III de Madrid, and Department of Economics, University of Maryland at College Park. The second author acknowledeges support from the following grants: Spanish MCyT BEC2002-00279, The European TMR-ERB-40618C97-0994 and the Secretaría de Estado de Universidades PR2003-0305. This paper was finished while Alvaro Escribano was visiting the Department of Economics, Georgetwon University, Washington DC, USA.
} 


\section{Introduction}

The properties of cointegration tests based on single equation error correction models (ECM test) are well known. The dependence of the critical values and the power of the ECM test on nuisance parameters is documented in Banerjee et al. (1986), Engle and Granger (1987), Kremers et al. (1992), and Banerjee et al. (1993) ${ }^{1}$.

The effects of having breaks when applying unit root test, like Dickey and Fuller (1979) test, etc., are also well known, see Stock (1994). Perron (1989) is a good starting point to see those impacts. From Clements and Hendry (1999), a structural break essentially corresponds to an intermittent shock with a permanent effect on the series. If this permanent shock is not explicitly taken into account, standard unit roots tests might mistake the structural break with a unit root. The results of Hendry and Neale (1990) and Perron and Vogelsang (1992) indicate that a neglected shift in the mean also leads to spurious unit roots. Rappoport and Reichlin (1989) is probably the first reference to check if we want to know the impact of having segmented trends as an alternative to a unit root model. Andrés et al. (1990) extended the analysis of Rappoport and Reichlin to more that one break point in the trend. Other references on breaks and unit roots tests are Banerjee et al. (1992); Zivot and Andrews (1992), and Leybourne et al. (1998).

From the applied point of view, the main nuisance is to add dummy variables for the structural breaks in order to apply valid unit root tests when the critical values obtained depend on the size and on the timing of the break. Again, the selection of the dummy variable is critical for the result of the test. A vast literature emerged searching for unknown break points using recursive or sequential tests (Andrews 1993; Andrews et al. 1996; Bai 1997; Vogelsang 1997; Bai and Perron 1998; Banerjee et al. 1998).

An important class of unusual events are additive outliers. These are events with a large, but temporary effect on the series. In certain cases, this effect dominates the remaining information contained in the series and biases unit root inference towards rejection of the unit root hypothesis even if the null hypothesis of a unit root is correct, as reported in Franses and Haldrup (1994) and Lucas (1995a,b).

With multiple time series the situation could be worse if the breaks are independent. Now we have to decide on the models that generate the anomalous observations (breaking trends, additive outliers, ... ) taking into account that those irregularities need not occur simultaneously nor on all of the variables. Therefore, the multivariate analysis is generally more difficult. However, in some cases it can be more simple if there is partial co-breaking in the series.

In empirical applications the addition of dummy variables to obtain parameter "constant" models is more the rule than the exception. The effects of including dummy variables to capture structural breaks in ECM tests have been previously analyzed by Campos et al. (1996). Once again, critical values (C.V.) depend on the particular type of dummy variable included in the model and is a nuisance for empirical applications

\footnotetext{
${ }^{1}$ See Arranz and Escribano (1998a,b, 2000) for a brief review of the main results.
} 
One alternative to avoid the use of dummy variables is to use robust estimation techniques. This is the approach taken by Lucas $(1995 \mathrm{a}, \mathrm{b})$ in the univariate case and Lucas (1997) and Franses and Lucas (1997a,b) in the multivariate case.

In this paper we follow a different route. The objective is to find robust modeling procedures to test for unit roots in the presence of additive outliers in an ECM context. Instead of including dummy variables in ECM models, we try to approximate those breaks by adding extra dynamic terms (lags), as determined by the SBIC criterion. In particular, we look at the critical values obtained with the overparameterized model and analyze the power of the ECM test, using Monte Carlo simulations. We also investigate whether the robustness properties of the ECM test improve by following the same steps not on the observable variables, but on the trend components obtained from trend-cycle decompositions, as in Arranz et al. (2000). In particular, we study three filters, the Hodrick and Prescott $(1980,1997)$ filter, the Baxter and King $(1995,1999)$ filter, HP and BK respectively from now on, and the median filter (see Wen and Zeng, 1999). Guay and St-Amant (1997) and Baxter and King (1995) provide some insights about the relationship between the HP and BK filters.

The structure of the paper is the following. In Sect. 2 we analyze the effects of having transitory breaks on alternative specifications of the ECM models, and in particular on the cointegrating relationship. Three types of co-breaking possibilities are studied in detail: simultaneous co-breaking, co-breaking in levels (not in differences) and co-breaking in differences (not in levels). We also study several cases without any co-breaking. Section 3 reviews the signal extraction filters that we apply. The trend component ECM models are introduced in Sect. 4. Section 5 presents the main results of the Monte Carlo simulation experiments. The usefulness of our approach is illustrated with an empirical application in Sect. 6. Finally, the conclusions and some comments for further research directions are included in Sect. 7.

\section{Error correction models with and without simultaneous co-breaking}

Consider the following conditional error correction model (ECM)

$$
\begin{aligned}
\Delta\left(y_{t}-\mu_{y, t}\right) & =a \Delta\left(z_{t}-\mu_{z, t}\right)+b\left[\left(y_{t-1}-\mu_{y, t-1}\right)-\alpha\left(z_{t-1}-\mu_{z, t-1}\right)\right]+u_{1 t} \\
\Delta\left(z_{t}-\mu_{z, t}\right) & =u_{2 t} .
\end{aligned}
$$

Assume that $\ldots, y_{-1}, y_{0}=0$ and $\ldots, z_{-1}, z_{0}=0$, let $\mu_{y, t}=\tilde{\mu}_{y, t}+s_{y} \delta_{t}^{y}$, $\mu_{z, t}=\tilde{\mu}_{z, t}+s_{z} \delta_{t}^{z}$, where $\delta_{t}^{y}$ and $\delta_{t}^{z}$ are iid Bernoulli variables independent of $u_{1, t}$ and $u_{2, t}$

$$
\left\{\begin{array}{l}
\operatorname{Pr}\left(\delta_{t}=1\right)=\operatorname{Pr}\left(\delta_{t}=-1\right)=\frac{\pi}{2} \\
\operatorname{Pr}\left(\delta_{t}=0\right)=1-\pi .
\end{array}\right.
$$

The terms $\tilde{\mu}_{y, t}$ and $\tilde{\mu}_{z, t}$ include all possible deterministic components like: constant terms, deterministic trends, dummy variables, segmented trends, etc. Define $B$ as the back-shift operator, $B^{k} y_{t}=y_{t-k}, \Delta=(1-B)$ is the first differencing operator, 
and let $(1,-\alpha)$ be the cointegrating vector. The stochastic errors $u_{1 t}$ and $u_{2 t}$ are jointly, and serially uncorrelated with zero mean, and constant variances, say $\sigma_{1}^{2}$ and $\sigma_{2}^{2}$, respectively.

Model (1a)-(1b) can be written in terms of the observable variables $y_{t}$ and $z_{t}$ as follows,

$$
\begin{aligned}
\Delta y_{t} & =c_{t}+a \Delta z_{t}+b\left(y_{t-1}-\alpha z_{t-1}\right)+u_{1 t} \\
\Delta z_{t} & =\Delta \mu_{z, t}+u_{2 t} \\
c_{t} & \equiv \Delta \mu_{y, t}-a \Delta \mu_{z, t}-b\left(\mu_{y, t-1}-\alpha \mu_{z, t-1}\right) \\
\mu_{z, t} & =\tilde{\mu}_{z, t}+s_{z} \delta_{t}^{z} \\
\mu_{y, t} & =\tilde{\mu}_{y, t}+s_{y} \delta_{t}^{y} .
\end{aligned}
$$

In this paper we investigate the effects of having alternative generating models (with partial co-breaks, etc.) for the stochastic intercept $c_{t}$ of (2a) given by (2c) on the ECM test for non-cointegration $(b=0)$.

Definition 1. We say that the time series $y_{t}$ and $z_{t}$ have co-breaks in levels if $\mu_{y, t}-\alpha \mu_{z, t}=c_{l}$, where $c_{l}$ is a finite constant parameter.

Definition 2. We say that the time series $y_{t}$ and $z_{t}$ have co-breaks in differences if $\Delta \mu_{y, t}-a \Delta \mu_{z, t}=c_{d}$, where $c_{d}$ is a finite constant parameter.

Definition 3. We say that the time series $y_{t}$ and $z_{t}$ have simultaneous co-breaks if $\Delta \mu_{y, t}-a \Delta \mu_{z, t}-b\left(\mu_{y, t}-\alpha \mu_{z, t}\right)=c_{s}$, where $c_{s}$ is a finite constant parameter.

From Definitions 1 and 2, it is clear that if $y_{t}$ and $z_{t}$ exhibit co-breaks in levels and in differences (full co-break), we have a particular case of simultaneous cobreaking.

In general, without having any co-break in levels or in differences, the most parsimonious representation in terms of unobservable variables is the conditional ECM model (1a). In terms of observable variables, the most parsimonious representation is $(2 \mathrm{a})$, because it only requires to add the regressors coming from the contemporaneous values of $c_{t}$. If we are interested in estimating the parameters $a, \alpha$ and $b$, we can estimate them by 1-step procedures (OLS or NLS) in ECM representation (2a), see Arranz and Escribano (2000) for details. However, to do that we need to know or to estimate $\mu_{y, t}$ and $\mu_{z, t}$, and this can incorporate arbitrary assumptions about unknown events (dummy variables, etc.), see Vogelsang (1999).

In this paper, we argue that in a non-stationary context $\mu_{y, t}$ and $\mu_{z, t}$ could include any possible combination of transitory breaks and outliers, which complicates the analysis. Therefore, we suggest to consider general detrending procedures to get rid of those unobserved non-stationary transitory elements. In particular, we recommend to specify error correction models in terms of the growth components variables (trend components) to obtain robust ECM test for non-cointegration $(b=0)$ in the presence of outliers, as will become clear in Sects. 4 and 5. 


\subsection{Error correction models under simultaneous co-breaking}

From Eqs. (2a)-(2c) and the analysis of Escribano (1987) and Andrés et al. (1990), it is clear that any error correction model in terms of the observable variables and constant parameters should account for the joint effects of the following elements: $\Delta \mu_{y, t}, \Delta \mu_{z, t}, \mu_{y, t-1}$ and $\mu_{z, t-1}$.

Previous error correction models with certain co-breaks have been treated in Campos et al. (1996) and Clements and Hendry (1999). In this section we study models with simultaneous co-breaks so that $c_{t}=0$. Under simultaneous co-breaks, (2a) and (2b) can be simplified to

$$
\begin{aligned}
& \Delta y_{t}=a \Delta z_{t}+b\left(y_{t-1}-\alpha z_{t-1}\right)+u_{1 t} \\
& \Delta z_{t}=\Delta \mu_{z, t}+u_{2, t} \\
& \mu_{z, t}=\tilde{\mu}_{z, t}+s_{z} \delta_{t}^{z}
\end{aligned}
$$

where (3a) has the form of the usual single equation error correction without a constant term since $c_{t}=0$. The parameter $s_{z}$ measures the size of the break, and $\delta_{t}^{z}$ is the additive outlier, see Sect. 5 for more details.

From Eqs. (3a) and (3b) it is clear that $y_{t} \sim I(1), z_{t} \sim I(1)$, and that they are cointegrated in the sense of Engle and Granger (1987) with cointegration vector equal to $(1,-\alpha)$ for certain parameter values of $b(-2<b<0)$. Notice that we are allowing for transitory breaks in the 'exogenous' variable $z_{t}$ that co-break simultaneously with the endogenous variables $y_{t}$ and therefore the breaks disappear from the conditional model, Eq. (3a).

\subsection{Alternative error correction models without simultaneous co-breaking}

In the previous section we have discussed the case of joint co-breaking in levels and in differences. Our purpose now is to discuss several interesting intermediate cases, see Arranz and Escribano (2000) for a full discussion.

We are considering several cases in the the simulation experiments later on: co-breaks in levels, but not in differences, co-breaks in differences, but in levels, and no co-breaks (independent breaks).

Case 1. Co-break in levels but not in differences. Co-break in levels $\left(\mu_{y, t}-\alpha \mu_{z, t}=\right.$ $c_{l}$ ). Taking first differences, we have $\Delta \mu_{y, t}-\alpha \Delta \mu_{z, t}=0$. But from Eq. (2c)

$$
c_{t}=\Delta \mu_{y, t}-a \Delta \mu_{z, t}-b c_{l}=(\alpha-a) \Delta \mu_{z, t}-b c_{l},
$$

and Eq. (2a) becomes

$$
\Delta y_{t}=-b c_{l}+(\alpha-a) \Delta \mu_{z, t}+a \Delta z_{t}+b\left(y_{t-1}-\alpha z_{t-1)}+u_{1, t}\right.
$$

From Eq. (2d), assuming $\tilde{\mu}_{z, t}=0$, we have

$$
\Delta y_{t}=-b c_{l}+(\alpha-a) s_{z} \Delta \delta_{t}^{z}+a \Delta z_{t}+b\left(y_{t-1}-\alpha z_{t-1}\right)+u_{1, t} .
$$

Therefore, under co-breaks in levels but not in differences, the breaks $\delta_{t}^{z}$ in the marginal process of $\Delta z_{t}$ affect the error correction model unless the COMFAC restriction is satisfied $(a=\alpha)$. Later on, we will analyze the effects of omitting the second term of the right-hand side of Eq. (6). 
Remark 1. co-break in levels $\Rightarrow$ co-break in differences if $a=\alpha$ (COMFAC restriction), for any value of $c_{t}$.

Case 2. Co-break in differences but not in levels. Co-break in differences: $\Delta \mu_{y, t}-$ $a \Delta \mu_{z, t}=c_{d}$ implies that $\Delta \mu_{y, t}-\alpha \Delta \mu_{z, t}=(a-\alpha) \Delta \mu_{z, t}+c_{d}$. From recursive substitution $\mu_{y, t}-\alpha \mu_{z, t}=\left(\mu_{y_{0}}-\alpha \mu_{z_{0}}\right)+c_{d} t+(a-\alpha) \mu_{z, t}$, and $c_{t}$ becomes

$$
c_{t}=c_{d}-b\left(\mu_{y, 0}-\alpha \mu_{z, 0}\right)-b c_{d}(t-1)-b(a-\alpha) \mu_{z_{t-1}}
$$

and Eq. (2a) can be written as

$$
\Delta y_{t}=c_{m}+b c_{d} t-b(a-\alpha) \mu_{z, t-1}+a \Delta z_{t}+b\left(y_{t-1}-\alpha z_{t-1)}+u_{1, t},\right.
$$

where $c_{m}$ is a constant equal to $c_{m}=c_{d}-b\left(\mu_{y, 0}-\alpha \mu_{z, 0}\right)+b c_{d}$. From Eq. (2d), assuming $\tilde{\mu}_{z, t}=0$,

$$
\Delta y_{t}=c_{m}+b c_{d} t-b(a-\alpha) s_{z} \delta_{t-1}^{z}+a \Delta z_{t}+b\left(y_{t-1}-\alpha z_{t-1)}+u_{1, t} .\right.
$$

Section 5 provides evidence of the effects of omitting the second and third terms of the right-hand side of (9)

Remark 2. Co-break in differences $\Rightarrow$ co-break in levels if $a=\alpha$ (COMFAC) and $c_{d}=0$. If $c_{d} \neq 0$ the regression (9) would need a trend.

Case 3. Independent breaks. The final possibility there is no co-breaking in levels nor in differences. This plausible empirical situation is the result of joining the effects of Eqs. (6) and (9). In our simulation study, we will consider independent breaks on $y_{t}$ and $z_{t}$, Eq. (10a), and also breaks on just one of the two series, Eqs. (10b) or (10c). Therefore, the term $c_{t}$ from (2c) will be one of the following:

$$
\begin{aligned}
& c_{t}=s_{y} \Delta \delta_{t}^{y}-a s_{z} \Delta \delta_{t}^{z}-b\left(s_{y} \delta_{t-1}^{y}-\alpha s_{z} \delta_{t-1}^{z}\right) \\
& c_{t}=-a s_{z} \Delta \delta_{t}^{z}+b \alpha s_{z} \delta_{t-1}^{z} \\
& c_{t}=s_{y} \Delta \delta_{t}^{y}+b s_{y} \delta_{t-1}^{y}
\end{aligned}
$$

and will analyze the misspecification impacts of considering $c_{t}$ as a constant term under alternative modeling strategies.

\section{Filters and signal extraction}

The usual aim of a filter in macroeconomic time series is to extract particular components of the series: trend, cycle, irregular, etc. In this paper, we are interested in splitting an observed $I(1)$ time series in two components,

$$
y_{t}=y_{t}^{g}+y_{t}^{c}
$$

where $y_{t}^{g}$ is the growth component and $y_{t}^{c}$ is the cyclical component. Two main alternatives are usually considered. First, define what the trend component is and hence the cycle will be the residual $\left(y_{t}-y_{g}^{g}\right)$.

$$
y_{t}=y_{t}^{g}+\left(y_{t}-y_{t}^{g}\right)
$$

Second, define the cycle and therefore the trend will be the residual $\left(y_{t}-y_{t}^{c}\right)$

$$
y_{t}=\left(y_{t}-y_{t}^{c}\right)+y_{t}^{c}
$$




\subsection{Baxter and King filter (BK)}

Most of the filters used with macroeconomic time series are two-sided infinite order moving averages, as pointed out by King and Rebelo (1993) and Baxter and King $(1995,1999)$. In practice the filter has to be approximated by a two-sided MA(k)

$$
y_{t}^{\star}=a_{0}+\sum_{h=1}^{k} a_{h}\left(B^{h}+B^{-h}\right) y_{t} .
$$

The implications of the filters are clearly seen in the frequency domain by looking at the frequency response function. The frequency response function of the two-sided $\operatorname{MA}(\infty)$ equation is given by

$$
\beta(\omega)=\sum_{h=-\infty}^{\infty} b_{h} e^{-i w h}
$$

while for the two-sided MA(k) the frequency response function is

$$
\alpha(\omega)=\sum_{h=-k}^{k} a_{h} e^{-i w h} .
$$

Baxter and King (1995) obtain optimal approximating filters by minimizing the mean square error of $\delta(\omega) \equiv \beta(\omega)-\alpha(\omega)$ with respect to $a_{h}$. The component $y^{c}$ could be obtained by applying trend reducing filters (high-pass filters) based on symmetric MA $(\mathrm{k})$ transformations. Baxter and King showed that when $\sum_{h=-k}^{k} a_{h}=0, y_{t}^{\star}$ has no trend if the growing component of $y_{t}$ was generated by deterministic trends (linear or quadratic) or by I(1) or I(2) processes.

Notice that the trend reduction condition, $\sum_{h=-k}^{k} a_{h}=0$, implies that the frequency response function, Eq. (14) satisfies $\alpha(0)=0$. The spectrum of $y_{t}^{c}$ is zero at the zero frequency and it is associated with the business cycle component $\left(y_{t}^{c}\right)$ and therefore $\left(y_{t}-y_{t}^{c}\right)$ is the trend component $\left(y_{t}^{g}\right)$. These trend reducing filters are called high-pass filters since they pass components of the data with frequency larger than a predetermined value $\underline{\omega}$ close to 0 . That is $\beta(\omega)=0$ for $|\omega|<\underline{\omega}$ and $\beta(\omega)=1$ for $|\omega| \geq \underline{\omega}$.

On the other hand, low-pass filters are determined so that $\beta(\omega)=0$ for $|\omega|>\underline{\omega}$ and $\beta(\omega)=1$ for $|\omega| \leq \underline{\omega}$ and therefore low frequencies, (long term movements) remain unchanged while others are canceled out. In terms of the finite symmetric MA(k) filter, this means that low-pass filters must satisfy $\sum_{h=-k}^{k} a_{h}=1$.

Baxter and King (1995) showed that an 'ideal' approximate low-pass filters could be obtained by choosing the coefficients of the two-sided MA(k) filter, equal to $a_{0}=\frac{1}{\pi} \underline{\omega}$ and $a_{h}=\frac{1}{h \pi} \sin (h w)$ for $h=1,2,3 \ldots$ Therefore, the complementary high-pass filter has coefficients $\left(1-a_{0}\right)$ at $h=0$ and $-a_{h}$ for $h=1,2,3, \ldots$

When the filter passes frequencies between $\underline{\omega}$ and $\bar{\omega}$ of the spectrum where $0<|\underline{\omega}|<|\bar{\omega}|<\pi$ it is called band-pass filter and can for example be obtained by subtracting two low-pass filters. Usually, the frequency interval is associated with the NBER business cycle duration as defined by Burns and Mitchell (1946) where $\underline{\omega}$ corresponds to 32 quarters ( 8 years) and $\bar{\omega}$ to 6 quarters (1.5 or 2 years). This low-pass filter is what we are calling the BK filter in the simulations. 


\subsection{Hodrick and Prescott filter (HP)}

The Hodrick and Prescott $(1980,1997)$ filter is widely used in macroeconomics to detrend series in order to study of the stylized facts of an economy along the business cycle. The basis of this filter is the following: starting from (11) they define the trend component as the solution to the following optimization problem

$$
\min _{\left\{y_{t}^{g}\right\}} \sum_{t=1}^{T}\left[\left(y_{t}-y_{t}^{g}\right)^{2}+\lambda\left(\Delta^{2} y_{t+1}^{g}\right)^{2}\right]
$$

The first term of (15) might be regarded as a measure of the goodness of fit of the trend component to the observed series, while the second one imposes a penalty in order to get a smooth trend component. The values of the parameter $\lambda$ suggested by Kydland and Prescott (1990) are $\lambda=1600$ for quarterly data and $\lambda=400$ for annual data, obtained as the ratio of the volatility of the irregular component relative to the volatility of the growth component.

Expressing the problem in terms of the backward shift operator, $B$, the decompositions is written as

$$
y_{t}=F(B)^{-1} y_{t}+C(B) y_{t}
$$

with $y_{t}^{g}=F(B)^{-1} y_{t}$ and $y_{t}^{c}=C(B) y_{t}$, where the polynomials in $B$ (filters) are $F(B)=\lambda B^{2}-4 \lambda B+(6 \lambda+1)-4 \lambda B^{-1}+\lambda B^{-2}=\lambda(1-B)^{2}\left(1-B^{-1}\right)^{2}+1$ and

$$
C(B)=\frac{\lambda(1-B)^{2}\left(1-B^{-1}\right)^{2}}{\lambda(1-B)^{2}\left(1-B^{-1}\right)^{2}+1},
$$

which is the HP filter. Notice that $F(1)=1$ and $C(1)=0$.

A number of authors have studied the basic properties of the HP filter, see for example Harvey and Jaeger (1993), King and Rebelo (1993), and Kaiser and Maravall (1999).

\subsection{The median filter}

The HP and BK filters are examples of linear filters. Furthermore, we will also consider a class of nonlinear filters, called the median filter (Wen and Zeng 1999), that has been proven to be very useful in recent years in signal processing. Median filters have two interesting properties: edge (sharp change) preservation and efficient noise attenuation with robustness against impulsive-type noise. Neither of these properties can be achieved by linear filtering techniques. To compute the output of a median filter, an odd number of sample values are sorted, and the middle or median value is used as the filter output. If the filter length is $2 n+1$, the filtering procedure is denoted as

$$
\operatorname{med}\left\{y_{t-n}, y_{t-n+1}, \ldots, y_{t}, \ldots, y_{t+n}\right\}
$$


Frequency analysis and impulse response have no meaning in median filtering since the impulse response of a median filter is zero. Nonetheless, a very important property of the median filter is the so-called root-convergence property, namely, any finite sample time series contains a root signal set that is invariant to the median filtering. From an economic point of view, this invariant property is of interest because it makes possible that possible structural shifts of economic fundamentals be not disturbed by the filtering operation. See Wen and Zeng (1999) for further details. This property is particularly relevant for our purpose, as we will notice in the Monte Carlo experiments.

\section{Trend components ECM test}

Decomposing the series $z_{t}$ as in (11) we get

$$
z_{t}=z_{t}^{g}+z_{t}^{c}
$$

If the actual series, $y_{t}$ and $z_{t}$, are $I(1)$ and $\left(y_{t}-\alpha z_{t}\right)$ is $I(0)$ they are cointegrated. In terms of the unobserved components we could write the cointegrating relationship as

$$
y_{t}-\alpha z_{t}=\left(y_{t}^{g}-\alpha z_{t}^{g}\right)+\left(y_{t}^{c}-\alpha z_{t}^{c}\right)
$$

Let $\bar{b}(B)$ be a general two-sided moving average filter where we impose some constraints in the $\bar{b}_{k}$ coefficients so that it is a low-pass filter (see Sect. 3.1 for details), and call $y_{t}^{g}=\bar{b}(B) y_{t}$ to the corresponding trend component. Then, multiplying Eq. (2a) by $\bar{b}(B)$ we get

$$
\Delta \bar{b}(B) y_{t}=\bar{b}(B) c_{t}+a \Delta \bar{b}(B) z_{t}+b\left[\bar{b}(B) y_{t-1}-\alpha \bar{b}(B) z_{t-1}\right]+\bar{b}(B) u_{1, t}
$$

which is an ECM model for the trend component

$$
\Delta y_{t}^{g}=c_{t}^{g}+a \Delta z_{t}^{g}+b\left[y_{t-1}^{g}-\alpha z_{t-1}^{g}\right]+\bar{b}(B) u_{1, t}
$$

Since $\bar{b}(B) u_{1, t}$ might have some autocorrelation, we can consider the ECM for the trend components with longer lags given by

$$
\phi_{y}(B) \Delta y_{t}^{g}=c_{t}^{g}+a \phi_{z}(B) \Delta z_{t}^{g}+b\left[y_{t-1}^{g}-\alpha z_{t-1}^{g}\right]+\eta_{t}
$$

where $\eta_{t}$ is considered white noise and the lags of $\phi_{y}(B) \Delta y_{t}^{g}$ and $\phi_{z}(B) \Delta z_{t}^{g}$ are determined by the SBIC criterion. We might expect that for significant smoothing, $c_{t}^{g}$ can be approximated by a constant or a linear trend.

Alternatively, from Eq. (2a) we can write the ECM model for a general trendcycle decomposition as,

$$
\Delta\left(y_{t}^{g}+y_{t}^{c}\right)=c_{t}+a \Delta\left(z_{t}^{g}+z_{t}^{c}\right)+b\left[\left(y_{t-1}^{g}+y_{t-1}^{c}\right)-\alpha\left(z_{t-1}^{g}+z_{t-1}^{c}\right)+u_{1, t}\right.
$$


and grouping terms, Eq. (23) can be written as

$$
\begin{aligned}
\Delta y_{t}^{g}= & c_{t}^{\star}+a \Delta z_{t}^{g}+b\left(y_{t-1}^{g}-\alpha z_{t-1}^{g}\right)+u_{1, t} \\
c_{t}^{\star}= & \left(\Delta \mu_{y, t}-\Delta y_{t}^{c}\right)-a\left(\Delta \mu_{z, t}-\Delta z_{t}^{c}\right) \\
& -b\left[\left(\mu_{y, t-1}-\alpha \mu_{z, t-1}\right)-\left(y_{t-1}^{c}-\alpha z_{t-1}^{c}\right)\right]
\end{aligned}
$$

In practice, since we do not know when the breaks occur, we would like to approximate (24a)-(24b) by

$$
\phi_{y}(B) \Delta y_{t}^{g}=c_{0}^{\star}+a \phi_{z}(B) \Delta z_{t}^{g}+b\left[y_{t-1}^{g}-\alpha z_{t-1}^{g}\right]+\epsilon_{t}
$$

The question of interest in this paper is whether the ECM test based on the t-ratio $\left(t_{\hat{b}}\right)$ of Eq. (25) is robust to the presence of outliers in the series.

\section{Monte Carlo simulation experiment}

Our data generating process (DGP) is based on several extensions of the one used by Kremers et al. (1992) and Campos et al. (1996). It is a linear first-order vector autoregression with normal disturbances, Granger causality in only one direction $(z \rightarrow y)$, and possible additive outliers in both variables $\left(y_{t}\right.$, and $\left.z_{t}\right)$ for the parameters of interest $a$ and $\alpha$.

\subsection{The model}

Our DGP is based on

$$
\begin{aligned}
\Delta y_{t} & =c_{t}+a \Delta z_{t}+b\left(y_{t-1}-\alpha z_{t-1}\right)+u_{1 t} \\
\Delta z_{t} & =\Delta \mu_{z, t}+u_{2 t} \\
c_{t} & =\Delta \mu_{y, t}-a \Delta \mu_{z, t}-b\left(\mu_{y, t-1}-\alpha \mu_{z, t-1}\right) \\
\mu_{z, t} & =\mu_{z, o}+s_{z} \delta_{j, t}^{z} \\
& \left\{\begin{array}{l}
\operatorname{Pr}\left(\delta_{t}^{z}=1\right)=\operatorname{Pr}\left(\delta_{t}^{z}=-1\right)=\frac{\pi}{2} \\
\operatorname{Pr}\left(\delta_{t}^{z}=0\right)=1-\pi
\end{array}\right.
\end{aligned}
$$

In order to get time series $y_{t}$ and $z_{t}$ with only co-breaks in differences, we impose that $\Delta \mu_{y, t}-a \Delta \mu_{z, t}=c_{d}=0.5$. On the other hand, to simulate a set of series with only co-breaks in levels, we impose $\Delta \mu_{y, t}-\alpha \Delta \mu_{z, t}=0$. The series $y_{t}$ and $z_{t}$ will show simultaneous co-breaking when $\Delta \mu_{y, t}-a \Delta \mu_{z, t}-b\left(\mu_{y, t-1}-\alpha \mu_{z, t-1}\right)=$ $c_{s}=0$. See Arranz and Escribano (1998b) for more details of the derivation. In the case of no co-breaks, we add another shock to $\mu_{y}$ given by

$$
\mu_{y, t}=\mu_{y, o}+s_{y} \delta_{j, t}^{y}
$$

where $\delta_{j, t}^{y}$ follows a stochastic process similar to $\delta_{j, t}^{z}$ in (26e) but mutually independent. 
Based on (26a)-(26d), the critical values $(b=0)$, and the power $(b \neq 0)$ of the test are obtained by Monte Carlo simulation experiments.

Without loss of generality, we take $\sigma_{1}^{2}=1, \alpha=1$ and $\sigma_{2}=s, \mu_{y, 0}=\mu_{z, 0}=0$. Thus, the experimental design variables are the parameters $a, b, s$, and the sample size, $T$. The experiment is a full factorial design with:

$$
\begin{aligned}
& a=0.0,0.5,1 \text { (contemporaneous correlation in first differences for } a \neq 0 \text { ) } \\
& b=0.0 \text { (no cointegration), } b<0 \text { (cointegration) } \\
& \pi=0,0.05,0.1 \text { (breaks for } \pi \neq 0 \text { ) } \\
& s=1,6,16 \text { (size of the breaks) } \\
& T=100,200,500,1000 \text { (sample sizes) }
\end{aligned}
$$

This represents 144 experiments for each value of $b$. Notice that when $a=1$ there is a common (COMFAC) restriction in the error correction model $(a=\alpha=$ $1)$.

The Monte Carlo experiments are based on 2000 replications of each experiment where the first 50 observations of the simulated series are dropped to consider random initial conditions.

To obtain the empirical critical values we simulate the $y_{t}$ and $z_{t}$ series following the DGP (26a)-(26e) with $b=0$ where we have imposed $\alpha=1$, and with those series we estimate the following three models

$$
\begin{aligned}
\Delta y_{t} & =c+a \Delta z_{t}+b\left(y_{t-1}-z_{t-1}\right)+u_{1 t} \\
\phi(B) \Delta y_{t} & =c+\theta(B) \Delta z_{t}+b\left(y_{t-1}-z_{t-1}\right)+u_{1 t} \\
\phi(B) \Delta y_{t}^{g} & =c+\theta(B) \Delta z_{t}^{g}+b\left(y_{t-1}^{g}-z_{t-1}^{g}\right)+u_{1 t}
\end{aligned}
$$

The order of the polynomials $\phi(B)$ and $\theta(B)$ are chosen by means of the SBIC criterion. The variables $y_{t}^{g}$ and $z_{t}^{g}$ are the trend components obtained by Hodrick Prescott filter (HP10 and HP100), Baxter and King filter (BK) and the median filter (MD). See also Sect. 3 for more details. The lower 5\% tail of the distribution of the $t(\hat{b})$ statistic is the empirical critical value considered. The empirical power of the test is calculated analogously by simulating the series with other parameter values of $b(b<0)$, and computing the percentage of rejections obtained using the previously obtained empirical critical values.

Notice that we avoid the case where $c_{d}=0$ because the critical values would be the ones obtained in the simultaneous co-breaking case, since under $b=0$ we have simultaneous co-breaking with $c_{s}=0$. Furthermore, notice that in the case where $a=\alpha=1$, co-breaks in differences would imply co-breaks in levels (full cobreaking). This comes from the fact that under the COMFAC restriction $(\alpha=a)$, co-breaks in levels implies co-breaks in differences (full co-break).

\subsection{ECM test based on the trend components: No outliers or no breaks}

Figure 1 represents the kernel density estimator of the whole empirical distribution of $t(\hat{b})$ for Model 2 (ECM test) and Model 3 (Filtered ECM test) using the HP10 filter, HP100 filter, BK and MD respectively. With all of the filters used we get 


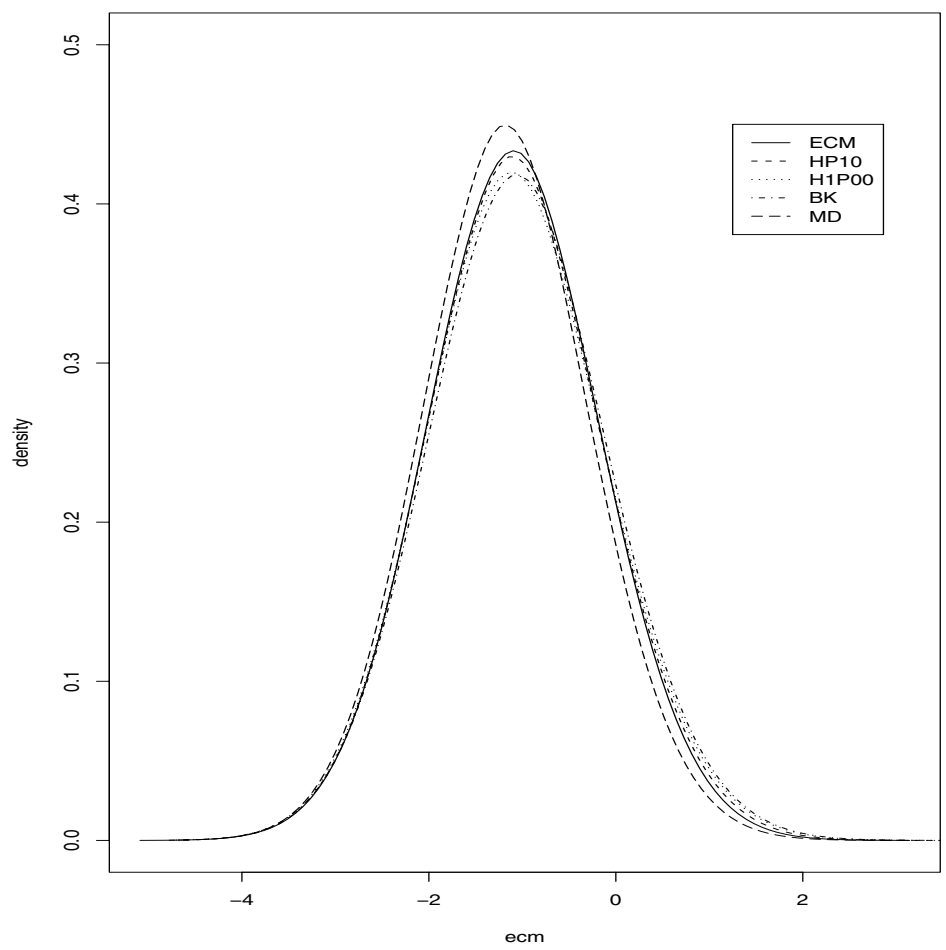

Fig. 1. Kernel density estimator of the ECM test statistic (Model 2), and Low-Pass filter ECM test (Model 3) under the null hypothesis. $T=1000, a=0$. No outliers

similar results. It is important to remark that the best results are obtained for the left tail of the distributions. This implies that the critical values used to test the null hypothesis of no-cointegration against the cointegration alternative are robust to the type of filter used, see Table 1.

In terms of power there is only a small loss when using the BK filter and even smaller when using the median filter (MA), see Fig. 2 and Table 2. The HP10 and HP100 filters display the lowest power.

\subsection{ECM test with additive outliers}

In order to evaluate the effects of the additive outliers on the ECM tests we evaluate the most favorable situation. That is, we allow for extra lags in the ECM models to approximate the effect of the outliers and run the ECM test based on Model 2 when the orders of the polynomial $\varphi(B)$ and $\theta(B)$ are chosen by the SBIC criterion.

From Fig. 3.1 we see the dramatic size distortions of having $10 \%$ contamination of additive outliers with independent shocks in $y_{t}$ and $z_{t}$ with Model 2, and it would be worse if we tested for non-cointegration $(b=0)$ on Model 1 instead. The problem 
Table 1. Critical value of the ECM tests (Models 1 and 2) and filtered ECM test (Model 3). No outliers $(\pi=0)$

\begin{tabular}{ccccc}
\hline$a$ & $\mathrm{~T}=100$ & $\mathrm{~T}=200$ & $\mathrm{~T}=500$ & $\mathrm{~T}=1000$ \\
\hline \multicolumn{5}{c}{ Model 1 } \\
0.0 & -2.624 & -2.518 & -2.654 & -2.639 \\
0.5 & -2.769 & -2.738 & -2.758 & -2.752 \\
1.0 & -2.875 & -2.842 & -2.878 & -2.929 \\
\multicolumn{5}{c}{ Model 2} \\
0.0 & -2.596 & -2.495 & -2.641 & -2.652 \\
0.5 & -2.801 & -2.787 & -2.754 & -2.789 \\
1.0 & -2.951 & -2.868 & -2.862 & -2.949 \\
\multicolumn{5}{c}{ Model 3. HP10 filter } \\
0.0 & -3.017 & -2.670 & -2.668 & -2.614 \\
0.5 & -3.272 & -3.071 & -2.859 & -2.874 \\
1.0 & -3.481 & -3.164 & -2.940 & -2.913 \\
\multicolumn{5}{c}{ Model 3. HP100 filter } \\
0.0 & -3.238 & -2.726 & -2.650 & -2.608 \\
0.5 & -3.707 & -3.071 & -2.832 & -2.797 \\
1.0 & -4.114 & -3.085 & -2.898 & -2.891 \\
\multicolumn{5}{c}{ Model 3. BK filter } \\
0.0 & -2.889 & -2.650 & -2.667 & -2.592 \\
0.5 & -3.069 & -2.750 & -2.676 & -2.752 \\
1.0 & -3.021 & -2.783 & -2.889 & -2.958 \\
0.0 & -2.749 & -2.616 & -2.654 & -2.664 \\
0.5 & -2.816 & -2.599 & -2.561 & -2.652 \\
1.0 & -2.859 & -2.715 & -2.736 & -2.778 \\
\hline \multicolumn{5}{c}{ Model 3. MD filter }
\end{tabular}

is that the whole distribution is shifted to the left and therefore we will detect too much cointegration. This result supports previous evidence given in Franses and Haldrup (1994).

However, the critical values (C.V.) obtained from Model 1 are very stable for the simultaneous co-breaking case and only marginally affected by the sample size $(T)$, the nuisance parameter $a$ and the size of the break $(s)$ Other partial co-breaking cases were analyzed in Arranz and Escribano (1998a); Arranz (2001).

ECM test from Model 3 with additive outliers. The testing model is

$$
\phi(B) \Delta y_{t}^{g}=c+\theta(B) \Delta z_{t}^{g}+b\left(y_{t-1}^{g}-z_{t-1}^{g}\right)+u_{1 t} \quad \text { (Model 3). }
$$

The intuition for expecting Model 3 to be a good approximation to the correctly specified model is the following. Equation (2a) is transformed into Eq. (24a) based on the trend components, where $c_{t}^{\star}$ is a stochastic intercept given by Eq. (24b). Since the additive outliers are transitory shocks they should mainly be part of the cycle in a trend-cycle decomposition. The stochastic slope $c_{t}^{\star}$ from (24b) should be 


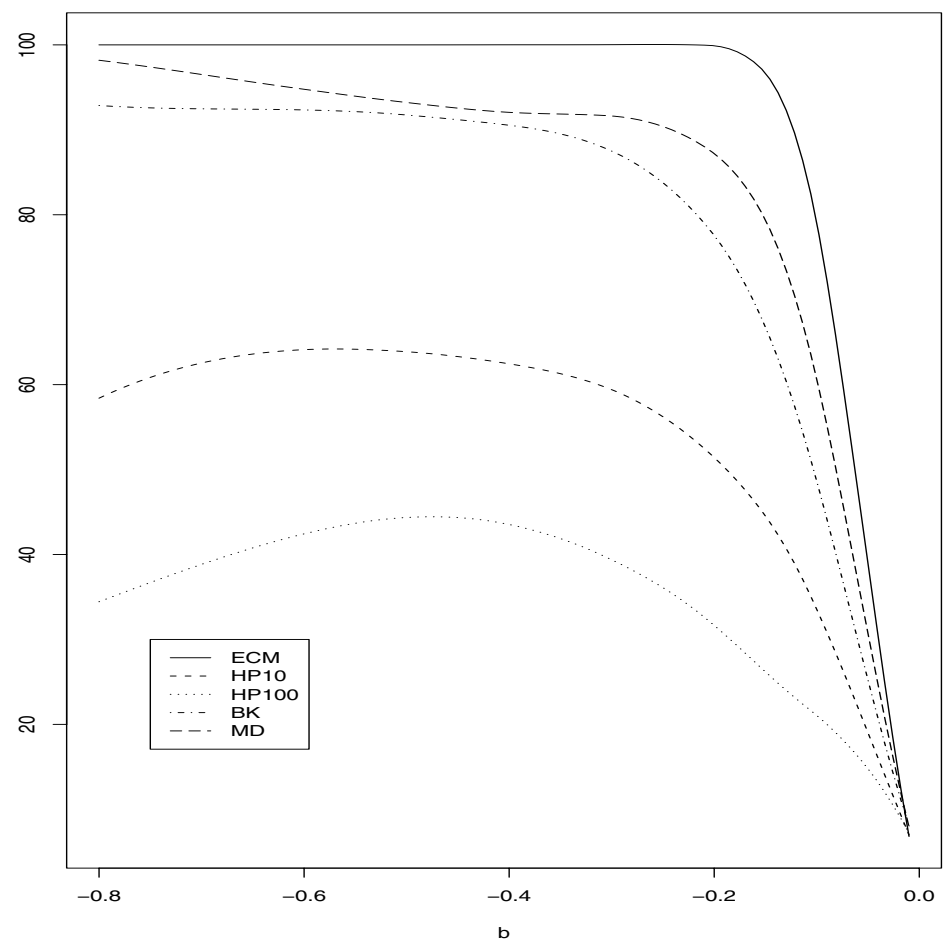

Fig. 2. Comparison of powers of the ECM test (Models 2 and 3). $T=100, a=0$. No outliers

a stationary series which can be approximated by lags of $\Delta y_{t}^{g}$ and $\Delta z_{t}^{g}$, and this is exactly what Model 3 does.

Tables 3-4 show the CV obtained for BK and MD filters under the six possible co-breaking and non-cobreaking situations previously analyzed. No matter what type of filter we use, the CV are stable for different sample sizes, different values of parameter $a$, different sizes of the jumps $(s)$. We also did the analysis for HP10, HP100, filters, see Arranz (2001). The only case where the CV of the ECM test depend seriously on the sample size $(T)$ is when there is only co-breaks in differences but not in levels. Notice, however the robustness of those critical values to nuisance parameters such as $s$ or $s$ (the jump size), even for a $10 \%$ of outliers $(\pi=0.1)$.

When we analyze the power of the test, we first observe that with co-breaks in differences not not in levels we get no power, as it happened with Model 2. Furthermore, the power of the test depends on the parameter $a$ in most cases. As expected, the power of the test based on Model 3 is lower than the one obtained with Model 2, but this is not true in all cases. In particular, in the case of having shocks only in the variable $y_{t}$, with $a=1$ and $s=1,16$, the most powerful test is the one based on Model 3 with the BK filter with $T=100$. In the case of having shocks in $z_{t}$, with $a=1$ the most powerful test for $T=100,200$ is the one based on the MD filter. Another feature of the test based on Model 3 and the MD filter 

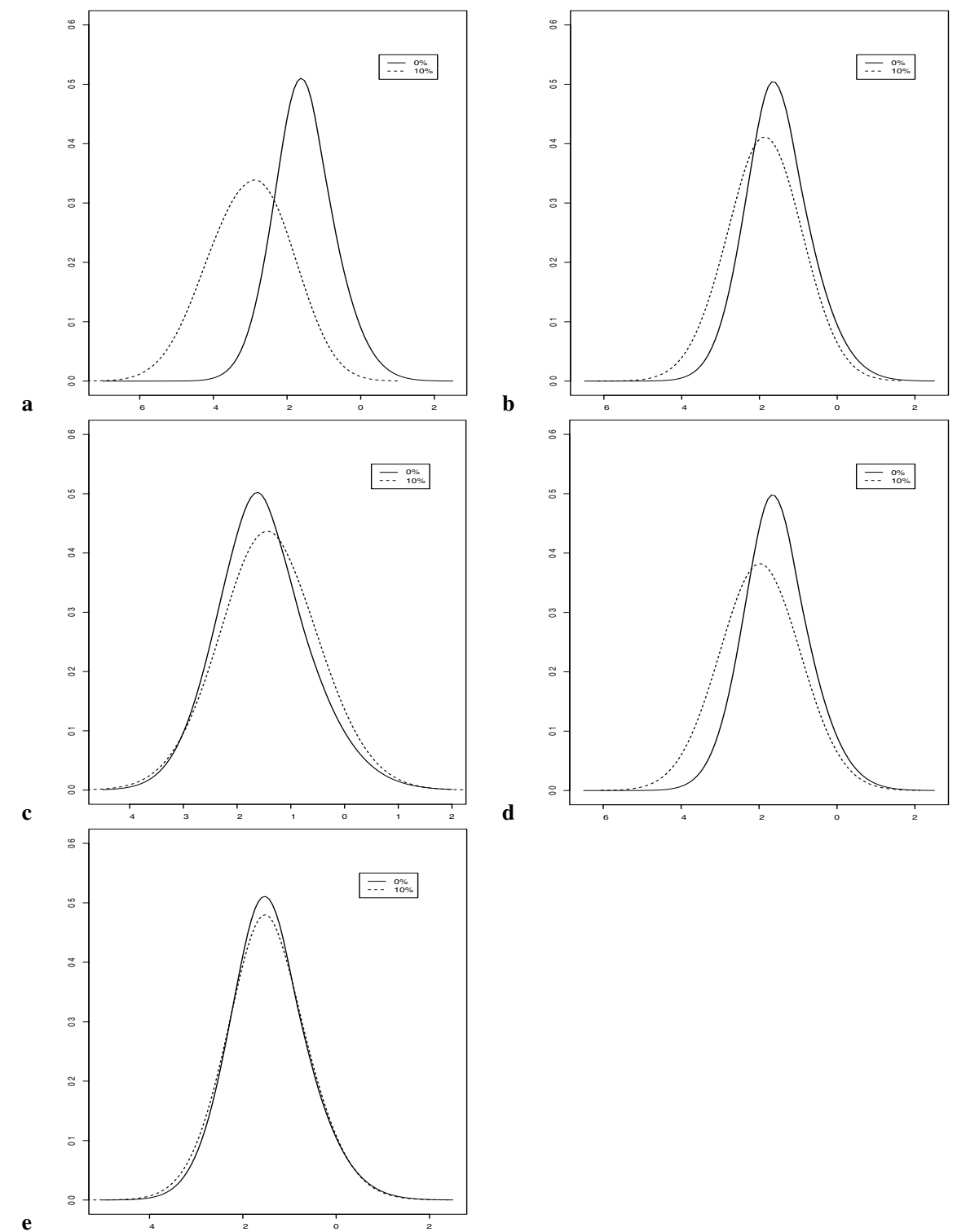

Fig. 3a-e. Kernel density estimator of the ECM test (Model 2) and the Low-pass filtered data test (Model 3) for (a) HP10, (b) HP100, (c) BK (d) and (e) MD filters. $T=1000, a=1$, independent shocks in variables $y_{t}$ and $z_{t}, s=16$ 
Table 2. Power of of the tests. $b=-0.2$. No outliers

\begin{tabular}{|c|c|c|c|c|}
\hline$a$ & $\mathrm{~T}=100$ & $\mathrm{~T}=200$ & $\mathrm{~T}=500$ & $\mathrm{~T}=1000$ \\
\hline \multicolumn{5}{|c|}{ Model 1} \\
\hline 0.0 & 99.45 & 100.00 & 100.00 & 100.00 \\
\hline 0.5 & 95.50 & 100.00 & 100.00 & 100.00 \\
\hline 1.0 & 87.75 & 100.00 & 100.00 & 100.00 \\
\hline \multicolumn{5}{|c|}{ Model 2} \\
\hline 0.0 & 99.15 & 100.00 & 100.00 & 100.00 \\
\hline 0.5 & 93.05 & 100.00 & 100.00 & 100.00 \\
\hline 1.0 & 79.70 & 99.95 & 100.00 & 100.00 \\
\hline \multicolumn{5}{|c|}{ Model 3. HP10 filter } \\
\hline 0.0 & 51.40 & 98.05 & 100.00 & 100.00 \\
\hline 0.5 & 30.15 & 76.75 & 100.00 & 100.00 \\
\hline 1.0 & 21.50 & 76.95 & 99.95 & 100.00 \\
\hline \multicolumn{5}{|c|}{ Model 3. HP100 filter } \\
\hline 0.0 & 31.75 & 81.05 & 100.00 & 100.00 \\
\hline 0.5 & 15.55 & 54.45 & 99.75 & 100.00 \\
\hline 1.0 & 11.25 & 42.80 & 99.25 & 100.00 \\
\hline \multicolumn{5}{|c|}{ Model 3. BK filter } \\
\hline 0.0 & 74.40 & 99.60 & 100.00 & 100.00 \\
\hline 0.5 & 46.15 & 92.85 & 100.00 & 100.00 \\
\hline 1.0 & 21.25 & 76.05 & 100.00 & 100.00 \\
\hline \multicolumn{5}{|c|}{ Model 3. MD filter } \\
\hline 0.0 & 88.10 & 99.65 & 100.00 & 100.00 \\
\hline 0.5 & 58.35 & 97.55 & 100.00 & 100.00 \\
\hline 1.0 & 30.60 & 85.00 & 100.00 & 100.00 \\
\hline
\end{tabular}

is that, apart from the case of co-breaks in differences but not in levels, the power of the test does not depend on the type of co-breaks considered. Furthermore, the test based on the MD filter yields the highest power among those tests based on Model 3.

\subsection{Robustness of critical values to the presence of outliers}

The question now is the following: Can we safely use the critical values of Table 1 to do cointegration tests, such as the ECM test, in the presence of additive outliers?

To answer this question we computed the empirical rejection frequencies obtained using the 5\% critical values of Table 1 when the data generating process given in Eqs. (26a)-(26f) is contaminated with the most dangerous case, that is, independent shocks in variables $y_{t}$ and $z_{t}$ under the null hypothesis $H_{0}: b=0$. If those critical values are robust, the percentage of rejections should be around 5\%.

Model 2 is clearly not robust since the percentage of rejection can reach $43.5 \%$ for a sample size of $T=100$ with $s=16$, see Fig. 3.a. Using Model 3 the results improve dramatically, see Figs. 3.a-e. With HP10 and BK the previous figure is reduced to $21.8 \%$ and $26.1 \%$, but with HP100 and MD those values are $5.1 \%$ and 
Table 3. Critical values. Model 3, BK filter, $\pi=0.1$

\begin{tabular}{|c|c|c|c|c|c|c|c|c|c|}
\hline \multirow[b]{2}{*}{$T$} & \multicolumn{3}{|c|}{$a=0.0$} & \multicolumn{3}{|c|}{$a=0.5$} & \multicolumn{3}{|c|}{$a=1.0$} \\
\hline & $s=1$ & $s=6$ & $s=16$ & $s=1$ & $s=6$ & $s=16$ & $s=1$ & $s=6$ & $s=16$ \\
\hline \multicolumn{10}{|c|}{ Simultaneous co-breaks } \\
\hline 100 & -2.843 & -2.712 & -2.680 & -3.073 & -2.766 & -2.682 & -2.889 & -2.812 & -2.752 \\
\hline 200 & -2.654 & -2.605 & -2.563 & -2.665 & -2.717 & -2.721 & -2.773 & -2.793 & -2.722 \\
\hline 500 & -2.652 & -2.682 & -2.588 & -2.670 & -2.792 & -2.832 & -2.883 & -2.927 & -2.928 \\
\hline 1000 & -2.589 & -2.566 & -2.556 & -2.752 & -2.882 & -2.887 & -2.961 & -3.044 & -3.050 \\
\hline \multicolumn{10}{|c|}{ Co-breaks in differences, not in levels } \\
\hline 100 & -2.471 & -2.450 & -2.416 & -2.504 & -2.474 & -2.465 & -2.487 & -2.496 & -2.480 \\
\hline 200 & -2.114 & -2.124 & -2.114 & -2.212 & -2.221 & -2.221 & -2.246 & -2.234 & -2.204 \\
\hline 500 & -1.962 & -1.937 & -1.929 & -2.015 & -1.991 & -1.905 & -1.985 & -1.949 & -1.896 \\
\hline 1000 & -1.854 & -1.854 & -1.853 & -1.880 & -1.764 & -1.626 & -1.755 & -1.654 & -1.624 \\
\hline \multicolumn{10}{|c|}{ Co-breaks in levels, not in differences } \\
\hline 100 & -2.890 & -2.797 & -2.784 & -2.990 & -2.796 & -2.728 & -2.889 & -2.812 & -2.752 \\
\hline 200 & -2.712 & -2.737 & -2.771 & -2.722 & -2.778 & -2.799 & -2.773 & -2.793 & -2.722 \\
\hline 500 & -2.686 & -2.944 & -2.932 & -2.740 & -2.894 & -2.909 & -2.883 & -2.927 & -2.928 \\
\hline 1000 & -2.603 & -2.999 & -3.028 & -2.837 & -3.044 & -3.111 & -2.961 & & -3.050 \\
\hline \multicolumn{10}{|c|}{ Independent shocks } \\
\hline 100 & -2.815 & -3.079 & -4.165 & -3.144 & -3.201 & -4.391 & -3.242 & -3.471 & -4.578 \\
\hline 200 & -2.637 & -2.720 & -4.015 & -2.868 & -2.934 & -4.553 & -2.749 & -3.033 & -4.901 \\
\hline 500 & -2.689 & -2.706 & -2.500 & -2.652 & -2.740 & -3.055 & -2.813 & -2.941 & -3.578 \\
\hline 1000 & -2.587 & -2.641 & -2.720 & -2.720 & -2.863 & -3.496 & -2.882 & -2.995 & -4.089 \\
\hline \multicolumn{10}{|c|}{ Shocks in $y_{t}$} \\
\hline 100 & -2.808 & -2.990 & -3.187 & -3.175 & -3.201 & -3.410 & -3.007 & -3.362 & -3.580 \\
\hline 200 & -2.629 & -2.536 & -2.938 & -2.783 & -2.770 & -3.329 & -2.770 & -2.959 & -3.539 \\
\hline 500 & -2.670 & -2.615 & -2.746 & -2.700 & -2.739 & -3.105 & -2.855 & -2.867 & -3.358 \\
\hline 1000 & -2.579 & -2.598 & -2.755 & -2.728 & -2.862 & -3.286 & -2.940 & -2.879 & -3.508 \\
\hline \multicolumn{10}{|c|}{ Shocks in $z_{t}$} \\
\hline 100 & -2.843 & -2.712 & -2.680 & -3.100 & -2.762 & -2.661 & -3.148 & -2.971 & -2.757 \\
\hline 200 & -2.654 & -2.605 & -2.563 & -2.783 & -2.608 & -2.532 & -2.767 & -2.877 & -2.756 \\
\hline 500 & -2.652 & -2.682 & -2.588 & -2.663 & -2.646 & -2.521 & -2.867 & -2.824 & -2.713 \\
\hline 1000 & -2.589 & -2.566 & -2.556 & -2.718 & -2.687 & -2.546 & -2.894 & -2.845 & -2.802 \\
\hline
\end{tabular}

$6.2 \%$, respectively, see Table 7. Recall that we only mention the results for the worst contamination types. For most of the parameter values analyzed all of the filters perform quite well, but the best one in terms of robustness and power is the MD filter.

It is unclear the reason of the bad performance of the HP filter. One possible reason is that we did apply the traditional version used in Macroeconomics, with fixed values of $\lambda$, which are not 'optimal'. The second reason might be that the effect of initial conditions on unit root tests (see Muller and Elliott, 1999) which is not addressed in our version of the filter, but might be solved in modified versions such as that of Kaiser and Maravall (1999), is worse in the case of the HP filter. However, there are also modified versions of both the BK and the MD filter which we are not using. 
Table 4. Critical values. Model 3, MD test, $\pi=0.1$

\begin{tabular}{|c|c|c|c|c|c|c|c|c|c|}
\hline \multirow[b]{2}{*}{$T$} & \multicolumn{3}{|c|}{$a=0.0$} & \multicolumn{3}{|c|}{$a=0.5$} & \multicolumn{3}{|c|}{$a=1.0$} \\
\hline & $s=1$ & $s=6$ & $s=16$ & $s=1$ & $s=6$ & $s=16$ & $s=1$ & $s=6$ & $s=16$ \\
\hline \multicolumn{10}{|c|}{ Simultaneous co-breaks } \\
\hline 100 & -2.775 & -2.813 & -2.813 & -2.810 & -2.873 & -2.850 & -2.816 & -2.729 & -2.733 \\
\hline 200 & -2.609 & -2.622 & -2.626 & -2.589 & -2.622 & -2.615 & -2.718 & -2.656 & -2.638 \\
\hline 500 & -2.701 & -2.658 & -2.667 & -2.580 & -2.591 & -2 & -2 . & -2.666 & -2.671 \\
\hline 1000 & -2.664 & -2.663 & -2.663 & -2.655 & -2.590 & & & -2.767 & -2.763 \\
\hline \multicolumn{10}{|c|}{ Co-breaks in differences, not in levels } \\
\hline 100 & -2.503 & -2.520 & -2.527 & -2.543 & -2.502 & -2.353 & -2.481 & -2.284 & -2.249 \\
\hline 200 & -2.1 & -2.174 & -2.1 & & -2 . & & & -2 & \\
\hline 500 & -1.9 & -1.980 & -1.975 & & -2.0 & & & -2 & -2 \\
\hline 1000 & -1.848 & -1.858 & -1.858 & -1.865 & -1.900 & -1.887 & & -1.934 & -1.902 \\
\hline \multicolumn{10}{|c|}{ Co-breaks in levels, not in differences } \\
\hline 100 & -2.831 & -2.727 & -2.727 & -2.835 & -2.853 & -2.850 & -2.816 & -2.729 & -2.733 \\
\hline 200 & -2.611 & -2.576 & -2.5 & -2 . & -2. & & & & -2 \\
\hline 500 & -2.655 & -2.599 & -2.623 & -2 & -2.6 & & & -2.666 & -2 \\
\hline 1000 & -2.621 & -2.538 & -2.538 & -2.651 & -2.600 & & & -2.767 & -2.763 \\
\hline \multicolumn{10}{|c|}{ Independent shocks } \\
\hline 100 & -2.789 & -2.769 & -2.769 & -2.845 & -2.942 & -2.942 & -2.876 & -3.078 & -3.070 \\
\hline 200 & -2.611 & -2.648 & -2.648 & -2. & -2.737 & -2.7 & -2.739 & -2.856 & -2.831 \\
\hline 500 & -2.68 & -2.641 & -2.641 & -2.5 & -2.645 & -2.666 & -2 & -2.802 & -2.770 \\
\hline 1000 & -2.657 & -2.634 & -2.634 & -2.668 & -2.713 & -2.713 & -2.829 & -2.855 & -2.854 \\
\hline \multicolumn{10}{|c|}{ Shocks in $y_{t}$} \\
\hline 100 & -2.801 & -2.760 & -2.760 & -2.818 & -2.875 & & & & \\
\hline 200 & -2.6 & -2.652 & -2.652 & -2 & -2.6 & -2 & & -2.767 & -2 \\
\hline 500 & -2.67 & -2.661 & -2.661 & -2.6 & -2.628 & -2 & & -2.744 & -2 \\
\hline 1000 & -2.644 & -2.632 & -2.632 & -2.664 & -2.696 & -2.696 & -2.808 & -2.807 & -2.793 \\
\hline \multicolumn{10}{|c|}{ Shocks in $z_{t}$} \\
\hline 100 & -2.775 & -2.813 & -2.813 & -2.828 & -2.849 & -2.854 & -2.8 & -2.956 & -2.956 \\
\hline 200 & -2.609 & -2.622 & -2.626 & & -2.684 & & & -2.831 & -2.831 \\
\hline 500 & -2.701 & -2.658 & -2.667 & -2.558 & -2.593 & -2.595 & -2.712 & -2.762 & -2.742 \\
\hline 1000 & -2.664 & -2.663 & -2.663 & -2.668 & -2.711 & -2.710 & -2.815 & -2.830 & -2.830 \\
\hline
\end{tabular}

\section{Empirical example}

In this section we illustrate the practical usefulness of our procedure by performing the analysis on annual observations of the CPI-based US/Finland real exchange rates during the period 1900-1988. This dataset has been previously studied in Perron and Vogelsang (1992); Franses and Haldrup (1994) and Franses and Lucas (1998). The question is whether the real exchange rate series during that period is stationary or not, i.e does the purchasing power parity (PPP) hold? We proceed by testing if the nominal exchange rates and the CPI ratio are cointegrated. First, we perform the analysis imposing the common factor restriction (COMFAC) with the following variables: $y_{t}$ is the log of the nominal exchange rate and $z_{t}$ is the log of CPI ratio. The cointegrating relationship is known and equal to the real exchange 
Table 5. Size adjusted power of the test. Model 3 BK test, $\pi=0.1, b=-0.2$

\begin{tabular}{|c|c|c|c|c|c|c|c|c|c|}
\hline \multirow[b]{2}{*}{$T$} & \multicolumn{3}{|c|}{$a=0.0$} & \multicolumn{3}{|c|}{$a=0.5$} & \multicolumn{3}{|c|}{$a=1.0$} \\
\hline & $s=1$ & $s=6$ & $s=16$ & $s=1$ & $s=6$ & $s=16$ & $s=1$ & $s=6$ & $s=16$ \\
\hline \multicolumn{10}{|c|}{ Simultaneous co-breaks } \\
\hline 100 & 76.65 & 83.15 & 94.90 & 44.55 & 51.55 & 73.70 & 24.30 & 24.15 & 26.40 \\
\hline 200 & 99.80 & 99.50 & 99.70 & 94.60 & 96.00 & 98.20 & 76.15 & 74.70 & 78.85 \\
\hline \multicolumn{10}{|c|}{ Co-breaks in differences, not in levels } \\
\hline 100 & 1.80 & 3.00 & 5.40 & 0.80 & 0.70 & 1.05 & 0.15 & 0.10 & 0.05 \\
\hline 200 & 1.35 & 3.50 & 6.15 & 0.20 & 0.15 & 0.80 & 0.00 & 0.00 & 0.00 \\
\hline 500 & 0.40 & 1.75 & 5.20 & 0.05 & 0.05 & 1.10 & 0.00 & 0.00 & 0.00 \\
\hline 1000 & 0.05 & 0.90 & 4.20 & 0.00 & 0.05 & 0.65 & 0.00 & 0.00 & 0.00 \\
\hline \multicolumn{10}{|c|}{ Co-breaks in levels, not in differences } \\
\hline 100 & 73.35 & 41.15 & 27.60 & 39.00 & 30.25 & 28.55 & 24.30 & 24.15 & 26.40 \\
\hline 200 & 99.60 & 92.70 & 82.70 & 92.25 & 84.35 & 77.00 & 76.15 & 74.70 & 78.85 \\
\hline \multicolumn{10}{|c|}{ Independent shocks } \\
\hline 100 & 77.70 & 77.75 & 27.30 & 43.50 & 47.90 & 9.65 & 28.25 & 19.00 & 3.65 \\
\hline 200 & 99.80 & 99.45 & 91.85 & 93.70 & 93.75 & 75.85 & 84.65 & 71.65 & 47.75 \\
\hline 500 & 100.00 & 100.00 & 100.00 & 100.00 & 100.00 & 99.00 & 100.00 & 99.45 & 80.40 \\
\hline \multicolumn{10}{|c|}{ Shocks in $y_{t}$} \\
\hline 100 & 78.35 & 83.40 & 98.15 & 45.45 & 62.95 & 97.10 & 26.45 & 66.00 & 96.25 \\
\hline 200 & 99.70 & 99.85 & 99.95 & 93.95 & 98.85 & 99.85 & 82.75 & 97.95 & 99.95 \\
\hline \multicolumn{10}{|c|}{ Shocks in $z_{t}$} \\
\hline 100 & 76.90 & 70.05 & 27.15 & 45.95 & 35.70 & 12.65 & 25.95 & 14.40 & 5.75 \\
\hline 200 & 99.85 & 99.25 & 79.45 & 93.20 & 85.00 & 40.50 & 80.60 & 50.95 & 13.90 \\
\hline 500 & 100.00 & 100.00 & 99.95 & 100.00 & 100.00 & 89.05 & 100.00 & 96.90 & 52.45 \\
\hline 1000 & 100.00 & 100.00 & 100.00 & 100.00 & 100.00 & 99.80 & 100.00 & 100.00 & 87.60 \\
\hline
\end{tabular}

rate, i.e. $r_{t}=y_{t}-z_{t}$. The number of lags in the ECM model is chosen by means of the SBIC criterion. From Granger's representation theorem (Engle and Granger 1987), if the variables are cointegrated the term $r_{t-1}$ should be significant in at least one of the two equations. We perform the ECM test by examining the $t$-stats of the coefficient corresponding to $r_{t-1}$ in both equations.

When the analysis is done on the observed variables, the values of the $t$-stats are -6.65 and -1.49 , when the dependent variables are $\Delta y_{t}$ and $\Delta z_{t}$, respectively. It is clear that the long run causality runs from $z_{t}$, the log of the CPI ratio, to $y_{t}$, the $\log$ of the nominal exchange rate, but it is unclear that the series are cointegrated due to the presence of additive outliers. From simulations of the DF and ADF test statistics, the critical values would indicate that the series are cointegrated in the cases of partial co-breaks and shocks only in $z_{t}$, the log of the CPI rate. However, it is not clear that the series are cointegrated if they have independent shocks or the shocks occur only in the variable $y_{t}$, the log of the nominal exchange rate, and this might well be the case. If we apply this procedure to the trend components ECM models obtained by the HP100 filter, the $t$-stats are -1.03 and -2.15 respectively, while with the BK filter the ECM test statistics are -1.84 and -0.47 , and finally -2.16 and -0.37 for the MD filter. As we can see from the critical values obtained from our simulations, the conclusion is that we cannot reject the null hypothesis 
Table 6. Size adjusted power of the test. Model 3 MD test, $\pi=0.1, b=-0.2$

\begin{tabular}{|c|c|c|c|c|c|c|c|c|c|}
\hline \multirow[b]{2}{*}{$T$} & \multicolumn{3}{|c|}{$a=0.0$} & \multicolumn{3}{|c|}{$a=0.5$} & \multicolumn{3}{|c|}{$a=1.0$} \\
\hline & $s=1$ & $s=6$ & $s=16$ & $s=1$ & $s=6$ & $s=16$ & $s=1$ & $s=6$ & $s=16$ \\
\hline \multicolumn{10}{|c|}{ Simultaneous co-breaks } \\
\hline 100 & 88.25 & 85.35 & 57.10 & 58.75 & 53.60 & 33.70 & 33.95 & 35.40 & 35.30 \\
\hline 200 & 99.70 & 99.75 & 98.10 & 97.80 & 97.65 & 94.65 & 85.80 & 89.10 & 89.75 \\
\hline \multicolumn{10}{|c|}{ Co-breaks in differences, not in levels } \\
\hline 100 & 3.20 & 3.35 & 3.25 & 1.25 & 1.55 & 1.10 & 0.50 & 0.35 & 0.25 \\
\hline 200 & 2.05 & 2.20 & 2.15 & 0.80 & 0.75 & 0.55 & 0.05 & 0.05 & 0.05 \\
\hline 500 & 0.30 & 0.35 & 0.35 & 0.05 & 0.20 & 0.15 & 0.00 & 0.00 & 0.00 \\
\hline 1000 & 0.05 & 0.00 & 0.00 & 0.00 & 0.00 & 0.00 & 0.00 & 0.00 & 0.00 \\
\hline \multicolumn{10}{|c|}{ Co-breaks in levels, not in differences } \\
\hline 100 & 86.90 & 86.60 & 86.50 & 56.45 & 54.25 & 54.25 & 33.95 & 35.40 & 35.30 \\
\hline 200 & 100.00 & 99.95 & 99.95 & 97.10 & 97.45 & 97.45 & 85.80 & 89.10 & 89.75 \\
\hline \multicolumn{10}{|c|}{ Independent shocks } \\
\hline 100 & 86.70 & 84.90 & 84.75 & 58.85 & 57.25 & 57.35 & 31.90 & 29.75 & 30.25 \\
\hline 200 & 99.70 & 99.60 & 99.65 & 97.05 & 94.90 & 94.95 & 85.05 & 80.20 & 81.60 \\
\hline \multicolumn{10}{|c|}{ Shocks in $y_{t}$} \\
\hline 100 & 87.05 & 87.25 & 87.25 & 59.60 & 57.60 & 57.80 & 30.70 & 32.00 & 32.15 \\
\hline 200 & 99.65 & 99.60 & 99.60 & 96.95 & 96.45 & 96.35 & 85.90 & 86.20 & 86.10 \\
\hline 500 & 100.00 & 100.00 & 100.00 & 100.00 & 100.00 & 100.00 & 99.95 & 99.95 & 99.95 \\
\hline \multicolumn{10}{|c|}{ Shocks in $z_{t}$} \\
\hline 100 & 88.30 & 86.30 & 86.25 & 57.10 & 59.05 & 58.65 & 31.15 & 30.75 & 30.90 \\
\hline 200 & 99.65 & 99.75 & 99.75 & 97.75 & 96.50 & 96.50 & 85.25 & 80.55 & 80.50 \\
\hline 500 & 100.00 & 100.00 & 100.00 & 100.00 & 100.00 & 100.00 & 100.00 & 99.95 & 99.95 \\
\hline
\end{tabular}

that the series are not cointegrated, which agrees with the findings in Franses and Haldrup (1994) and Franses and Lucas (1998).

Notice, however, that imposing the COMFAC restriction reduces the power of the ECM test, see Kremers et al. (1992). Therefore, we apply the tests without imposing the COMFAC restriction, as we did in Sect. 5. In the case of the BK filter, the ECM test statistics are -3.63 and -0.86 , and -6.71 and -1.42 for the MD filter. Since the absolute value of the second statistic is not significant, it suggests that the second variable is weakly exogenous for the long run parameter of interest. However, from the first statistic, we conclude that the series are cointegrated, using the critical values from our Monte Carlo experiments, and that the real exchange rate follows a stationary process with outliers. This conclusion is consistent with the result of Vogelsang (1999) and Arranz et al. (2000).

\section{Conclusions}

In this paper we have analyzed the effects of having additive outliers in a multivariate context with cointegrated variables. Previous evidence shows that usual non-cointegration tests (like ECM tests) tend to find too much cointegration in 
Table 7. Robustness against outliers. Rejection frequencies when the model is simulated with DGP (26a)-(26f) with independent shocks in variables $y_{t}$ and $z_{t}$ with $\pi=0.1$ under $b=0$, using critical values obtained without outliers

\begin{tabular}{|c|c|c|c|c|c|c|c|c|c|}
\hline & \multicolumn{3}{|c|}{$a=0.0$} & \multicolumn{3}{|c|}{$a=0.5$} & \multicolumn{3}{|c|}{$a=1.0$} \\
\hline$T$ & $s=1$ & $s=6$ & $s=16$ & $s=1$ & $s=6$ & $s=16$ & $s=1$ & $s=6$ & $s=16$ \\
\hline \multicolumn{10}{|c|}{ Model 2} \\
\hline 100 & 5.35 & 6.30 & 13.95 & 7.55 & 17.55 & 16.45 & 10.05 & 38.90 & 19.25 \\
\hline 200 & 6.05 & 7.75 & 30.85 & 7.00 & 19.40 & 37.45 & 10.95 & 43.10 & 50.15 \\
\hline 500 & 5.05 & 3.25 & 1.25 & 6.65 & 16.35 & 10.75 & 7.35 & 34.80 & 33.30 \\
\hline 1000 & 5.35 & 3.40 & 3.60 & 6.45 & 16.80 & 20.45 & 5.50 & 21.75 & 43.55 \\
\hline \multicolumn{10}{|c|}{ Model 3, HP1O } \\
\hline 100 & 4.75 & 3.45 & 2.50 & 4.95 & 2.00 & 2.00 & 4.15 & 1.65 & 2.05 \\
\hline 200 & 5.60 & 4.35 & 4.90 & 3.75 & 1.95 & 4.05 & 4.75 & 2.15 & 4.55 \\
\hline 500 & 5.00 & 4.90 & 7.20 & 4.70 & 2.95 & 9.35 & 6.10 & 5.00 & 15.40 \\
\hline 1000 & 5.10 & 6.00 & 11.00 & 4.75 & 3.35 & 13.80 & 5.50 & 5.40 & 21.85 \\
\hline \multicolumn{10}{|c|}{ Model 3, HP100 } \\
\hline 100 & 5.85 & 3.70 & 1.95 & 5.05 & 1.70 & 0.65 & 4.50 & 1.55 & 0.40 \\
\hline 200 & 5.60 & 5.15 & 4.35 & 5.00 & 3.90 & 2.20 & 7.10 & 6.75 & 3.70 \\
\hline 500 & 5.30 & 5.35 & 5.20 & 6.40 & 3.50 & 3.40 & 6.65 & 5.10 & 3.85 \\
\hline 1000 & 5.35 & 4.95 & 5.35 & 6.05 & 4.15 & 4.45 & 6.15 & 5.00 & 5.15 \\
\hline \multicolumn{10}{|c|}{ Model 3, BK filter } \\
\hline 100 & 3.95 & 7.20 & 34.75 & 5.60 & 7.00 & 41.10 & 6.65 & 10.00 & 48.55 \\
\hline 200 & 4.65 & 5.80 & 22.15 & 5.90 & 6.85 & 34.15 & 4.65 & 9.35 & 45.70 \\
\hline 500 & 5.05 & 5.25 & 3.75 & 4.80 & 5.80 & 11.35 & 3.85 & 5.55 & 18.65 \\
\hline 1000 & 4.85 & 5.85 & 7.50 & 4.65 & 5.85 & 16.70 & 4.00 & 5.50 & 26.10 \\
\hline \multicolumn{10}{|c|}{ Model 3, MD filter } \\
\hline 100 & 5.55 & 5.25 & 5.25 & 5.35 & 6.50 & 6.50 & 5.10 & 7.60 & 7.60 \\
\hline 200 & 4.90 & 5.20 & 5.20 & 5.20 & 6.30 & 6.25 & 5.25 & 6.40 & 6.40 \\
\hline 500 & 5.15 & 4.90 & 4.90 & 5.15 & 6.25 & 6.30 & 4.90 & 5.80 & 5.50 \\
\hline 1000 & 4.85 & 4.35 & 4.35 & 5.25 & 5.90 & 5.90 & 5.40 & 6.15 & 6.25 \\
\hline
\end{tabular}

this case. The problem is partially solved by using overparameterized models that include extra lags of the regression variables.

We have analyzed the different effects of the additive outliers in a multivariate context, covering cases from simultaneous co-breaks, partial co-breaks to independent shocks in each of the variables of the model. Obviously, the worst distortion is obtained in the case of independent shocks.

We suggest to approach this problem by doing the non-cointegration test on the ECM models based on the trend component instead of on the usual ECM model with the observed variables. We have analyzed by Monte Carlo simulation experiments different trend-cycle decompositions based on the Hodrick-Prescott filter (HP10, HP100), the Baxter and King filter (BK) and the median filter (MD). Most of them yield good results in terms of robustness, but the best ones are the HP100 and MD filters. On the other hand, in terms of power, the best results are provided by the BK and MD filters. Our results suggest that more attention should be paid to nonlinear filters such as the median (MD) filter. 
The empirical methodology is applied to CPI-based US/Finland real exchange rates. The data set is particularly relevant since there are conflicting conclusions obtained in the literature depending on the test used. On the one hand, Franses and Haldrup (1994) and Franses and Lucas (1998) found evidence of no cointegration while on the other hand, Vogelsang (1999) found evidence of cointegration. Our conclusions support that the series are cointegrated if we use the most powerful version of the test and find no evidence of cointegration if we impose the COMFAC restriction, explaining the reason for having conflicting empirical results. Our procedure is robust to the presence of additive outliers and does not require prior identification of the outliers.

\section{References}

Andrés, J., Escribano, A., Molinas, C., Taguas, D. (1990) La Inversión en España: Econometría con Restricciones de Equilibrio. A. Bosch

Andrews, D. (1993) Tests of Parameter Instability and Structural Change with Unknown Break Point. Econometrica 61: 821-856

Andrews, D., Lee, W., Ploberger, W. (1996) Optimal Changepoints Tests for Normal Linear Regression. Journal of Econometrics 70: 9-38

Arranz, M. (2001) Unit Root and Cointegration with Additive Outliers and Structural Breaks: Small Sample Solutions. PhD thesis, Universidad Complutense de Madrid

Arranz, M., Escribano, A. (1998a) Cointegration Testing under Transitory Breaks: Robust ECM Testing Procedures. Working Paper 98-82, Universidad Carlos III de Madrid

Arranz, M., Escribano, A. (1998b) Detrending Procedures and Cointegration Testing: ECM Tests under Structural Breaks. Working Paper 98-15, Universidad Carlos III de Madrid

Arranz, M., Escribano, A. (2000) Cointegration Testing under Structural Breaks: A Robust Extended Error Correction Model. Oxford Bulletin of Economics and Statistics 61: 23-52

Arranz, M., Escribano, A., Marmol, F. (2000) Effects of Applying Linear and Nonlinear Filters on Tests for Unit Roots with Additive Outliers. Working paper, Department of Statistics and Econometrics, Universidad Carlos III de Madrid

Bai, J. (1997) Estimating Multiple Breaks one at a Time. Econometric Theory 13: 315-352

Bai, J., Perron, P. (1998) Estimating and Testing Linear Models with Multiple Structural Changes. Econometrica 66 : 47-78

Banerjee, A., Dolado, J., Hendry, D., Smith, G. (1986) Exploring Equilibrium Relationships in Econometrics through Static Models: Some Monte Carlo Evidence. Oxford Bulletin of Economics and Statistics 48: 853-877

Banerjee, A., Dolado, J., Galbraith, J.W., Hendry, D.F. (1993) Cointegration, Error-Correction, and the Econometric Analysis of Non-Stationary Data. Oxford University Press, Oxford

Banerjee, A., Lumsdaine, R., Stock, J. (1992) Recursive and Sequential Tests of Unit Root and Trend Break Hypothesis: Theory and International Evidence. Journal of Business and Economic Statistics 10: 271-287

Banerjee, A., Lazarova, S., Urga, G. (1998) Bootstrapping Sequential Tests for Multiple Structural Breaks. Discussion Paper 17-98, Center for Economic Forecasting, London Business School

Baxter, M., King, R. (1995) Measuring Business Cycles: Approximate Band-Pass Filters for Economic Time Series. Working Paper 5022, NBER

Baxter, M., King, R. (1999) Measuring Business Cycles: Approximate Band-Pass Filters for Economic Time Series. Review of Economics and Statistics 81: 575-593

Burns, A., Mitchell, W. (1946) Measuring Business Cycles, NBER

Campos, J., Ericsson, N., Hendry, D. (1996) Cointegration Tests in the Presence of Structural Breaks. Journal of Econometrics 70: 187-220

Clements, M., Hendry, D. (1999) Forecasting Non-stationary Economic Time Series. MIT Press, Chapt 9 
Dickey, D., Fuller, W. (1979) Distribution of the estimators for autoregressive time series with a unit root. Journal of the American Statistical Association 74: 427-431

Engle, R.F., Granger, C.W.J. (1987) Cointegration and Error-Correction: Representation, Estimation, and Testing. Econometrica 55: 251-276

Escribano, A. (1987) Co-Integration, Time Co-Trends and Error-Correction Systems: An Alternative Approach. Discussion Paper 8715, CORE

Franses, P., Lucas, A. (1997a) Outlier Robust Cointegration Analysis of Dutch Interest Rates. In: Proceedings of the 1996 Meeting of the American Statistical Association. ASA, pp. 106-109

Franses, P., Lucas, A. (1997b) Outlier Robust Cointegration Tests. VU Research Memorandum 97-45, Vrije Universiteit Amsterdam

Franses, P., Lucas, A. (1998) Outlier Detection in Cointegration Analysis. Journal of Business and Economic Statistics 16: 459-468

Franses, P., Haldrup, N. (1994) The Effects of Additive Outliers on Unit Roots and Cointegration. Journal of Business and Economic Statistics 12: 471-478

Guay, A., St-Amant, P. (1997) Do the Hodrick-Prescott and Baxter-King Filters Provide a Good Approximation of Business Cycles? Cahiers de Recherche 53, CREFE

Harvey, A.C., Jaeger, A. (1993) Detrending, Business Cycles Facts and the Business Cycle. Journal of Applied Econometrics 8: 231-47

Hendry, D., Neale, A. (1990) The Impact of Structural Breaks on Unit Root Tests. In; Hackl, P., Westlung, A. (eds.) Economic Structural Change: Analysis and Forecasting. IIASA and Springer, Berlin Heidelberg New York, pp. 95-119

Hodrick, R., Prescott, E. (1980) Post-war U.S. Business Cycle: An Empirical Investigation. Technical report, Carnegie-Mellon University

Hodrick, R., Prescott, E. (1997) Post-war U.S. Business Cycle: An Empirical Investigation. Journal of Money, Credit and Banking 29: 1-16

Kaiser, R., Maravall, A. (1999) Estimation of the Business Cycle: A Modified Hodrick-Prescott Filter. Spanish Economic Review 1: 175-206

King, R., Rebelo, S. (1993) Low Frequency Filtering and Real Business Cycles. Journal of Economic Dynamics and Control 17: 207-31

Kremers, J., Ericsson, N., Dolado, J. (1992) The Power of Cointegration Tests. Oxford Bulletin of Economics and Statistics 54: 349-67

Kydland, F., Prescott, E.C. (1990) Business Cycles: Real Facts and a Monetary Myth. Federal Reserve Bank of Minneapolis Quarterly Review 14: 3-18

Leybourne, S., Mills, T., Newbold, P. (1998) Spurious Rejections by Dickey-Fuller Tests in the Presence of a Break Under the Null. Journal of Econometrics 87: 191-203

Lucas, A., 1995a) An Outlier Robust Unit Root Tests with an Application to the Extended Nelson-Plosser Data. Journal of Econometrics 66: 153-173

Lucas, A., 1995b) Unit Roots Tests based on M Estimators. Econometric Theory 11: 331-346

Lucas, A. (1997) Cointegration Testing Using Pseudo Likelihood-Ratio Tests. Econometric Theory 13: $149-169$

Muller, U., Elliott, G. (1999) Tests for Unit Roots and the Initial Observation. Discussion Paper 2001-19, Department of Economics, UCSD

Perron, P. (1989) The Great Crash, the Oil Price Shock and the Unit Root Hypothesis. Econometrica 57, 1361-1401

Perron, P., T. Vogelsang (1992) Nonstationarity and Level Shifts with an Application to Purchasing Power Parity. Journal of Business and Economic Statistics 10: 301-320

Rappoport, P., Reichlin, L. (1989) Segmented Trends and Nonstationary Time Series. Economic Journal 99: $168-177$

Stock, J. (1994) Unit Roots, Structural Breaks and Trends. In: Engle, R.F., McFadden, D.L. (eds.) Handbook of Econometrics, Vol. 4. North-Holland, Amsterdam, Chapt. 46, pp. 2270-2381

Vogelsang, T. (1997) Wald-Type Tests for Detecting Breaks in the Trend of a Dynamic Time Series. Econometric Theory 13: 818-849

Vogelsang, T. (1999) Two Simple Procedures for Testing for a Unit Root when there are Additive Outliers. Journal of Time Series Analysis 20: 173-192 
Wen, Y., Zeng, B. (1999) A Simple Nonlinear Filter for Economic Time Series Analysis. Economics Letters 64: 151-160

Zivot, E., Andrews, D. (1992) Further Evidence on the Great Crash, the Oil Price Shock and the Unit Root Hypothesis. Journal of Business and Economic Statistics 10: 251-270 